\title{
Género, crisis económica y fragilidad financiera: desenredando la madeja ${ }^{1}$
}

\author{
Alicia GIRÓN* \\ Instituto de Investigaciones Económicas \\ Universidad Nacional Autónoma de México \\ alicia@servidor.unam.mx
}

Recibido: 12.10 .2011

Aceptado: 10.11.2011

\section{RESUMEN}

El presente artículo intenta, desde una perspectiva feminista, abordar la gran transformación que a partir del ciclo económico han tenido las mujeres desde la posguerra hasta la actualidad. En este periodo, las recurrentes crisis económicas responden a cambios estructurales del sistema capitalista donde la búsqueda de la rentabilidad ha permeado el mercado laboral con rostro femenino. Se intenta demostrar cómo el cambio estructural a nivel mundial ha influido en la relación existente entre la macroeconomía, la mesoeconomía y la microeconomía. Las relaciones entre el género y los procesos de la esfera de la producción se han visto envueltos en una crisis profunda que muestra la necesidad de incidir en la forma de cómo se ha producido a lo largo de varios decenios frente a la profundidad de la recesión económica internacional.

Palabras clave: Género, crisis económica, fragilidad financiera, mercado femenino.

\section{Gender, economic crisis and financial fragility}

\begin{abstract}
The present article approaches from a feminist perspective, the great transformation women have had in the economic cycle from postwar period to the present time. Recurrent economic crises in this period reply to structural changes of the capitalist system where the search of the profitability has permeated the labor market with a feminine face. It attempts to show how the structural change worldwide has influenced the existing relation between macroeconomics, mesoeconomics and microeconomics. The relationships between gender and the processes of the sphere of production are surrounded in a deep crisis that shows the need to affect the way the system has produced against the depth of the international economic recession
\end{abstract}

Key words: Gender, economic crisis, financial fragility, female market.

1 Una primera discusión del presente trabajo se llevó acabo en el Seminario Internacional "Equidad de Género y Cambio Social en América Latina y el Caribe” organizado por el Grupo de Trabajo: Feminismo y cambio en América Latina y el Caribe del Consejo Latinoamericano de Ciencias Sociales [CLACSO] en el Banco Central de Venezuela en julio de 2011. Se agraden las observaciones de Silva Berger de FLACSO, Argentina; Ma. Luisa González Marín, investigadora, y Vianney Fernández Villagómez, becaria, del Instituto de Investigaciones Económicas [IIEc] de la Universidad Nacional Autónoma de México [UNAM], México.

* Coordinadora del Grupo de Trabajo Género, Globalización y Desarrollo de CLACSO durante 20072009. Investigadora del Instituto de Investigaciones Económicas IIEc, tutora del Posgrado en Estudios Latinoamericanos y del Posgrado de Economía de la UNAM. Miembro de la Academia de Ciencias y de la Academia de Economía Política en México. Dirección postal: Circuito Mario de la Cueva s/n Ciudad de la Investigación en Humanidades. Ciudad Universitaria, Copilco El Ato, Coyoacán, Distrito Federal. MÉXICO, CP04510. Blog: www.aliciagiron.com 
"La mujer ha superado en gran parte la distancia que la separa del hombre por medio del trabajo, el único que puede garantizar una libertad concreta. En el momento en que deja de ser un parásito, el sistema fundado sobre su independencia se derrumba; entre ella y el universo ya no resulta necesario un mediador masculino. La maldición que pesa sobre la mujer vasalla consiste en que no se le permite actuar, por lo que se obsesiona en la imposible persecución del ser a través del narcisismo, del amor y la religión. Productiva y activa, tiene noción de su trascendencia. En sus proyectos se afirma concretamente como sujeto, por medio de su relación con el objetivo que persigue, con el dinero y los derechos que conquista, da pruebas de su responsabilidad. Muchas mujeres tienen conciencia de sus condiciones, incluso aquellas que ejercen los oficios más modestos.”

Simone de Beauvoir: "La Mujer Independiente” en El Segundo Sexo: Los hechos y los mitos.

\section{INTRODUCCIÓN²}

Uno de los retos más importantes es profundizar hasta qué punto la teoría feminista y la economía como ciencia social pueden explicar las relaciones que existen entre el género, la crisis financiera y la fragilidad económica en el desenvolvimiento del proceso de acumulación durante los últimos setenta años. La relación entre el género y la fragilidad económica, en el curso de la presente investigación, se convierte en una pregunta clave que merece abordarse desde varias aristas y perspectivas. ¿Cómo se puede articular desde una visión de género el impacto que han tenido las crisis económicas recurrentes y la crisis económica en curso en un terreno de fragilidad financiera constante?

En este trabajo, al tomar en cuenta la complejidad del proceso por el que está atravesando la economía internacional, sólo se intenta desenredar la madeja desde una perspectiva heterodoxa entre los conceptos enunciados en el título del presente artículo. Por lo cual, el rostro femenino del mercado laboral, más que una posible emancipación de las mujeres por lograr equidad en el trabajo, fue la necesaria absorción de ellas en el proceso de acumulación, lo que permitió la irrupción masiva de las mujeres en la fuerza de trabajo no sólo para absorber una parte del ejército industrial de reserva sino para disminuir los salarios y elevar la rentabilidad del capital.

\footnotetext{
2 Una primera discusión del presente trabajo se llevó acabo en el Seminario Internacional "Equidad de Género y Cambio Social en América Latina y el Caribe” organizado por el Grupo de Trabajo: Feminismo y cambio en América Latina y el Caribe del Consejo Latinoamericano de Ciencias Sociales [CLACSO] en el Banco Central de Venezuela en julio de 2011. Se agraden las observaciones de Silva Berger de FLACSO, Argentina; Ma. Luisa González Marín, investigadora, y Vianney Fernández Villagómez, becaria, del Instituto de Investigaciones Económicas [IIEc] de la Universidad Nacional Autónoma de México [UNAM], México.
} 
El objetivo de este trabajo es plantear desde la perspectiva de la relación entre género, fragilidad económica y crisis financiera los cambios del desenvolvimiento de las mujeres a través de las esferas de la producción y circulación. No sólo se toca la importancia de observar las grandes transformaciones en las políticas macroeconómicas a partir de la posguerra, sino también en cómo las decisiones de los grandes organismos financieros transformaron el entorno de las mujeres al interior de los hogares y las familias, resultado de las políticas contraccionistas que impactaron en la disminución del empleo.

Hoy, la visión de la economía de género, desde una perspectiva transdisciplinaria y multidisciplinaria, presenta una realidad muy compleja. Hilar la problemática y sentar en la mesa del debate lo complejo del tema es el reto que se presenta a continuación.

\section{EL SIGLO XX, LA TEORÍA FEMINISTA Y EL “NEOLIBERALISMO”}

El siglo XX se caracterizó por tres grandes revoluciones. La gran revolución socialista, que dividió al mundo global en dos modos de producción divergentes y asimétricos; la revolución de la teoría económica keynesiana y la revolución feminista, que transformó los valores de la sociedad patriarcal. Desde una perspectiva de género, las tres grandes revoluciones se entrelazan a través de las esferas de la producción y circulación incidiendo en la superestructura del Estado. La igualdad de la mujer pasa a ser fundamental tanto en el socialismo para incrementar la productividad como en el capitalismo para aumentar los ingresos familiares y el consumo en los hogares. Las mujeres vienen a ser creadoras de riqueza monetaria a partir de su incorporación al trabajo remunerado y participan como consumidoras al incentivar la demanda y el consumo del proceso económico desde la perspectiva keynesiana.

En este apartado, es importante resaltar la reflexión que señala Berger (2010) basándose en Nancy Frazer en la subterránea afinidad entre feminismo y "neoliberalismo". Por un lado, el feminismo aboga por la equidad de género e igualdad de condiciones en el mercado laboral frente a la autoridad tradicional androcentrista y, por el otro lado, la familia patriarcal limita la participación de las mujeres como fuerza de trabajo libre para disminuir los salarios, aumentar el ejército de reserva e incrementar los ingresos del hogar.

"Efectivamente, la familia patriarcal limita el desarrollo del mercado de trabajo. Si estalla la familia se amplia el mundo de las mercancías. En el "neoliberalismo" estas dos críticas de la autoridad tradicional, la feminista y la neoliberal parecen converger" (Berger, 2010:132).

$\mathrm{Al}$ observar los datos del incremento de las mujeres como jefas de hogar y proveedoras del ingreso familiar, se justifica la tendencia de una mayor participación 
en el mercado laboral a nivel internacional. No por ello el "neoliberalismo" significa "igualdad". Por lo cuál, el punto de convergencia entre feminismo y "neoliberalismo" es justo la inserción de las mujeres en el mercado de trabajo luchando por la equidad en los salarios y por una mayor participación en las decisiones de carácter económico, político y social. El "neoliberalismo" se basa en el trabajo de las mujeres y su cada vez mayor participación en el mercado laboral para aumentar las desigualdades de los ingresos.

La teoría feminista ha planteado nuevos elementos a partir de la participación de las mujeres en un contexto de fragilidad, inestabilidad y eclosión económica. El impacto en las mujeres en un proceso de recurrentes crisis económicas y su inserción cada vez más como parte de la fuerza laboral para incrementar los ingresos familiares ha transformado no sólo los patrones familiares sino los procesos económicos, políticos y sociales. Este proceso de grandes transformaciones, desde una perspectiva económica feminista, cuestiona el debate en torno a cómo las mujeres en calidad de sujetos económicos fueron resultados de los cambios estructurales del proceso de acumulación capitalista durante el siglo pasado. Las necesidades del "neoliberalismo" por incorporar a las mujeres a la economía monetaria crearon un discurso de emancipación. Es así como el crédito juega un elemento fundamental para la emancipación de las mujeres, bandera del progreso económico y del empoderamiento económico, político y social.

\section{CLASES SOCIALES, GÉNERO Y FAMILIA}

Existe una relación estrecha entre clase social y género. No es lo mismo, las mujeres en el área urbano-rural y las mujeres de clase media urbana frente a las mujeres que hasta la fecha subsisten en un ambiente de relaciones precapitalista o de economías no monetarias. El capitalismo y la propia crisis se sustentan en el grado de explotación de las mujeres no sólo como ejército de reserva, sino también en la búsqueda de mejores condiciones de desarrollo. La crisis económica, si bien impacta de manera generalizada a toda la economía y la sociedad, en la realidad existe diferencias entre los sectores económicos afectados y los renglones en donde se ubican las mujeres dependiendo de la clase social y el nivel de acuerdo a la escala de la distribución de los ingresos. Las mujeres y el feminismo tienen un rostro diferente según la clase social a la que pertenecen. No por ello, las relaciones del patriarcado que la sociedad y la cultura han transmitido a lo largo de la historia, permanecen en las diferentes clases sociales y niveles de ingreso.

Sólo para sustentar estos enunciados, es importante realizar un rápido recuento de los cambios significativos que permiten que la mujer de clase media urbana entre con mayor facilidad al mercado de trabajo. Un ejemplo son los cambios en la cocina que se dan al interior de los hogares en el periodo previo a la Segunda Guerra Mundial en los Estados Unidos. Estos cambios facilitaron la expansión de la industria y la posibilidad de un segundo salario, por supuesto menor al del hom- 
bre, como parte del ingreso familiar. Las grandes transformaciones en la cocina, lugar tradicional de lo femenino, facilitaron mejores condiciones de emancipación del trabajo doméstico para las mujeres de clase media en los Estados Unidos y en el resto de las sociedades de otros países que llevaron un proceso industrial. Esto permitió que las mujeres accedieran a ser proveedoras del ingreso familiar.

En la clase urbana baja, las mujeres se insertaron en la economía formal, muchas siguieron en la economía informal, pero no dejaron de cumplir con la jornada familiar. Las mujeres, sin dejar la economía del cuidado, las tareas de la casa y otras tareas que por su condición de ser mujer le son arbitrariamente atribuidas por la sociedad, siguieron cumpliendo con los valores impuestos por la sociedad patriarcal. La mayoría de estas mujeres son ejemplo de la triple jornada. No obstante, muchas de las mujeres se quedaron rezagadas del desarrollo económico ligado al proceso industrial.

En el área rural, las mujeres campesinas no sólo han sido parte de la esfera de la producción con tareas definidas en la división social del trabajo tanto al interior del núcleo familiar como por las tradiciones y valores culturales de regiones donde impera el poder patriarcal. En la transformación de economías no monetarias a economías monetarias, las mujeres, a través del microcrédito, se convirtieron en sujetos económicos rentables de las microfinancieras. Una visión "neoliberal” de ubicarlas en sectores productivos donde la rentabilidad del sector financiero se amplía a través de los circuitos financieros. Las políticas financieras que han utilizado el microcrédito para las mujeres nacen principalmente como programas prioritarios del Banco Mundial y de otros organismos financieros internacionales como son los programas del Banco Interamericano de Desarrollo (BID) para América Latina.

El rostro de las familias de clase media hicieron del "american way of life" una tendencia a seguir como parte de la imagen de una sociedad desarrollada desde los años treinta hasta antes del rompimiento de los Acuerdos de Bretton Woods (1971). Característica del siglo XX, la familia de clase media fue un valuarte del desarrollo. En este entorno es donde principalmente las hijas de estas familias lograron escalar desde los niveles de la educación primaria, pasando por la instrucción media y hasta la universidad una preparación equivalente y a veces superior al hombre lo que permitió que hubiera cada vez mejores mujeres preparadas para el mercado de trabajo e incidir en la disminución de los salarios a nivel general para aumentar los ingresos y ganancias de los grandes corporativos internacionales así como de las empresas pequeñas y medianas de carácter nacional. A partir de la década de los setenta, la incorporación de las mujeres aumentó a grandes saltos en los países de mediano desarrollo.

El modelo de la familia tradicional cambió drásticamente a través de tres generaciones. Hay una gran transformación entre las abuelas que nacieron entre la segunda y tercera década del siglo $\mathrm{XX}$, las hijas que nacieron antes y después de la mitad del siglo y las nietas que nacieron entre los setentas y ochentas. Hoy, al 
inicio del siglo XXI, las niñas que están por nacer, mujeres del futuro, se enfrentan a luchas feministas muy diferentes a cien años antes. Indudablemente que [Se puede reemplazar "que" por una coma] la revolución de las mujeres en el mercado laboral permitió mejorar los ingresos familiares y cambiaron el mercado laboral tanto en el sector formal como en el sector informal. Ellas representaron una fuerza económica y política que incidió en las pautas de consumo y en las decisiones de la familia, la empresa y de la política nacional.

\section{MUJERES EN UN ENTORNO GLOBAL DE CRISIS RECURRENTES}

Hay dos trabajos que ayudan a profundizar, desde una perspectiva feminista, los grandes cambios que han tenido las mujeres en una sociedad patriarcal en el proceso de acumulación capitalista. "Los circuitos de la crisis: una perspectiva feminista” de Girón (2010) y "Género y Crisis Económica” de Pearson y Sweetman (2011). Girón enfatiza que:

“...la complejidad de la crisis que estamos viviendo en estos momentos implica no sólo hacer una reflexión del proceso de desarrollo planteado por las instituciones de Bretton Woods durante los últimos setenta años sino también la forma en que ha evolucionado el proceso de producción en todas sus vertientes” (Girón, 2010:29).

Para Pearson y Sweetman, gran parte de los estudios se han centrado en el epicentro de la crisis financiera dejando de lado la esfera de la producción y reproducción donde las mujeres han sido permeadas por el entorno del cambio estructural (Pearson y Sweetman 2011: 1).
"En nuestros días, no sólo es, la crisis financiera cuya gran rapidez se viene des- envolviendo desde finales del 2006 y en el transcurso del 2007-2012 sino las com- plicaciones entretejidas con el cambio climático, los efectos en el medio ambiente y la crisis de los alimentos. Es en este laberinto de los circuitos de la crisis donde la profundización de las desigualdades entre hombres y mujeres aflora con mayor evidencia en sus relaciones con la producción, la circulación y el dinero”.

La gran crisis del 2007, más profunda que la crisis de 1929, ha estado precedidas por crisis recurrentes, el reforzamiento de inversionistas institucionales, un sistema financiero paralelo y un Estado minimalista. Esta crisis es parte del ciclo que se inicia en 1971 y continúa con el fin de la banca de inversión y la quiebra de Lehman Brothers en 2008. La crisis va acompañada de cambios en la dinámica del capitalismo en la esfera financiera afectando diferentes regiones monetarias. Pero también, fortaleciéndose en países emergentes y creando la base de futuras crisis. Es una crisis de largo alcance y de largo plazo. El núcleo del origen de la crisis radica en la esfera productiva. Es la estructura de la producción lo que ha transformado las pautas de trabajo, una mayor inserción de las mujeres como fuerza de tra- 
bajo y una expansión de grandes inversiones de los países desarrollados a las hoy economías emergentes.

Las mujeres se han insertado en el mercado laboral a grandes saltos a raíz de las crisis económicas recurrentes durante las últimas cuatro décadas. No obstante, que las políticas macroeconómicas cuyas medidas contraccionistas son las que han impactado en la disminución de los puestos de trabajo y los salarios a nivel familiar, las mujeres se han preparado para cubrir los puestos de trabajo tanto en la economía formal como informal. La participación de las mujeres en los países latinoamericanos y asiáticos, a raíz de la inestabilidad y los constantes planes de estabilización en la región propiciaron una penetración masiva de las mujeres. Más que una lucha por la emancipación y la igualdad, las mujeres formaron parte de las necesidades del cambio estructural y la división internacional del trabajo en el contexto internacional. La igualdad quedó en el tintero de la ley.

Las mujeres, durante el periodo comprendido entre los años setenta y la primera década del presente siglo, han sido sujetos sociales expresión de grandes transformaciones de nuestro tiempo. Periodo considerado como un laboratorio por excelencia para estudiar a las mujeres desde una perspectiva feminista. Pero también, la mujer como sujeto social, ha incidido en la necesidad no sólo de transformar las políticas públicas con enfoque de género sino también en el arco iris de líneas de investigación difícilmente abordadas en años anteriores.

El empoderamiento de las mujeres en la política y en los parlamentos es un hecho real así como la participación cada vez mayor en los puestos de decisión de las grandes empresas del sector público y privado y los equipos de carácter público de los estados. El Informe Brecha de Género (World Economic Forum, 2007) muestra las oportunidades que han tenido las mujeres en las decisiones de carácter político a través de la vida democrática. Es muy importante observar el indicador de la brecha de género porque para romper la brecha es importante el papel de las políticas públicas en dar mejores oportunidades a las mujeres basadas en la educación, la salud y alimentación.

\section{MACROECONOMÍA, MESOECONOMÍA Y MICROECONOMÍA}

El debate del desenvolvimiento de las mujeres en el curso de la crisis destaca puntos importantes. Por lo cuál el análisis a partir de las políticas macroeconómicas, mesoeconómicas y microeconómicas desde una visión de género plantean nuevos retos para la construcción de un mundo mejor.

Para Elson (2011), el origen de la crisis nace en la esfera financiera afectando la esfera económica - precisamente- donde se producen los bienes y servicios. Por tanto, en la producción y circulación el empleo y el ingreso han sido afectados. Es en la esfera de la reproducción, donde el trabajo y el capital se reproducen a través 
de las diferentes generaciones. A diferencia de Elson, en el trabajo de Girón (2010), se sostiene la hipótesis de que son los cambios estructurales en el sistema capitalista los que se involucran en mutaciones en los procesos de la producción y circulación desde antes de que se exprese la crisis financiera. Por tanto, es en la esfera de la producción y circulación donde estos dos circuitos se entrelazan con el circuito del género.

La crisis se origina en la esfera económica y no en la esfera financiera, produciendo grandes transformaciones en el mercado laboral. No olvidemos, la tesis de Crotty (2000) donde describe el fin de los años dorados y el nacimiento del régimen neoliberal. En su tesis, este autor enfatiza que el descenso de la tasa de ganancia en la década de los setenta en los Estados Unidos origina un cambio estructural a nivel mundial. El “... bajo crecimiento y tasas de ganancias bajas en la industria que estimula los flujos de capital hacia países menos desarrollados” (Crotty, 2000: 16). El "régimen neoliberal” traerá en consecuencia ante las crisis recurrentes políticas contraccionistas; un sistema financiero paralelo bajo la conducción de un Estado minimalista; el Banco Central deja de ser empleador de última instancia ${ }^{3}$ para convertirse en prestamista de última instancia de los bancos comerciales públicos y privados; y, por último, quizás el más importante, el crédito deja de ser una función social para el desarrollo y la equidad.

Por tanto, el entrelazamiento entre los cambios ocurridos en el marco de la macroeconomía permean indiscutiblemente los cambios en el sujeto femenino. En los hogares las decisiones del sujeto femenino como participante en el mercado laboral, proveedora del ingreso familiar y promotora de la demanda agregada cobran fuerza a partir de la década de los setenta.

El siguiente esquema se ha elaborado para sostener la relación entre las decisiones macroeconómicas y su impacto en los hogares y la vida cotidiana de las mujeres. Este esquema es muy importante para poder entender el proceso de los cambios en el curso del ciclo económico y los periodos de crisis y eclosión en las diferentes esferas del proceso de acumulación de capital. La incidencia que tienen las decisiones de los organismos financieros internacionales y los bancos centrales en el movimiento de los flujos de capital a nivel macroeconómico a través de las políticas de tasas de interés y de las políticas financieras están de común acuerdo con los intereses de los grandes conglomerados financieros y comerciales. Por un lado, el destino de los flujos de capital y las grandes ganancias de los corporativos; por el otro, la disminución de la ayuda oficial y la profundización de las políticas contraccionistas inciden en los procesos migratorios. Es un hecho que las políticas

\footnotetext{
3 La visión heterodoxa del banco central como prestamista de última instancia no termina solamente ahí sino que es la institución por medio de la cuál hay un monopolio monetario cuya autoridad es ejercida por el Estado moderno. Es responsable del circulante monetario y de enfrentar las quiebras bancarias. Pero más allá de su función principal el banco central a través de su política monetaria es el empleador de última instancia. El banco central crea empleo a través de la política monetaria.
} 
fiscales, monetarias y financieras implementadas por el Banco Central han reorientando los movimientos de la fuerza laboral a nivel internacional.

Los países que ocupan los primeros lugares en la recepción de remesas son de donde provienen cada vez más mujeres que se dedican a las actividades relacionadas con la economía del cuidado en los países donde encuentran empleo. Las transformaciones del panorama social de algunas regiones, resultado de las políticas descritas, afloran en la esfera de la microeconomía donde se expresan los cambios que impactan el diario acontecer de los ciudadanos: hombres, mujeres, niños y niñas. El periódico Financial Times recientemente presentó el mapa de los países emisores y receptores de los flujos de capital correspondientes a las remesas durante los últimos años (Financial Times, 2011). La recepción de remesas se ha agravado en el curso de la crisis actual afectando a las familias receptoras. La economía del cuidado en los países europeos y en Estados Unidos se ha restringido a pesar de la informalidad en que muchas mujeres trabajan en este sector por el aumento del desempleo en los países del Norte.

Otro renglón, sin duda importante, es la volatilidad del precio de los commodities. El modelo primario-exportador en que los países latinoamericanos han profundizado su dependencia del mercado mundial, al dejar de lado el modelo de sustitución de importaciones, le da a las decisiones de política económica un grado de fragilidad muy alto. Es decir, la disminución de las divisas amplia o restringe el gasto público de los gobiernos al depender de las divisas de los productos de exportación. Países emergentes como Argentina, Brasil y México siguen políticas al igual que el resto de los países latinoamericanos respetando la estabilidad del déficit público. Hasta el momento, es difícil explicar cómo las reservas de los bancos centrales siguen aumentando y en su caso sólo sirven para apreciar el tipo de cambio y mantener economías abiertas sin lograr invertir esos ingresos en el corto plazo. La abstinencia de invertir las reservas en proyecto productivos está poniendo en peligro inversiones a través del gasto social en el futuro del bienestar de la gran mayoría de la población.

La segunda división del cuadro presentado consiste en analizar la mesoeconomía. Es decir, cómo el Estado confronta el espacio de la macroeconomía y lo suministra a partir del excedente de su producción vía el gasto público. En lenguaje keynesiano estaríamos partiendo del multiplicador a través del gasto público que incentiva el gasto privado, incide en mayores créditos para los pequeños y medianos empresarios y, por tanto, estaría aumentando el financiamiento para el desarrollo del país. Al aumentar los ingresos y la capacidad productiva del país el empleo pasa a ser parte del círculo virtuoso del crecimiento y del desarrollo. No obstante, este círculo virtuoso que se presenta a partir de políticas expansivas implementadas por el Banco Central, en la dinámica actual de la crisis, se confunde con políticas contraccionistas donde se castra la función del prestamista de última instancia. En vez de que el Banco Central procure y sea el empleador de última instancia provoca al poner techos a la deuda pública y al gasto público una dinámica de contrac- 
ción del empleo y disminución del ingreso. En este proceso, la siguiente esfera que corresponde a la microeconomía de los hogares y las familias se ve influenciada por la dinámica presentada en la macroeconomía y la mesoeconomía.

En esta esfera de la microeconomía es importante no restar fuerza a cómo la disminución del gasto público incide en el aumento de la pobreza al disminuir los gastos en salud y educación pública. La brecha entre las clases sociales de menores ingresos se amplía no obstante los programas sociales para el alivio de la pobreza. Estos programas son de dos tipos. Primero “...consiste en la realización de acciones de complemento del ingreso familiar mediante subsidios 0 , la segunda, que apunta a la generación de empleo mediante programas públicos” (Pardo, 2003). El impacto que han tenido las políticas de estabilización en los países ha incidido en actividades que cobran más fuerza para el caso de las mujeres. Por ejemplo, la economía del cuidado cobra gran fuerza por el trabajo que realizan la mayoría de las mujeres de los países andinos que han emigrado a países donde un alto porcentaje de la población ha envejecido como es el caso de España e Italia. Incluso en los Estados Unidos las mujeres mexicanas y centroamericanas cumplen un papel importante dentro de las actividades del cuidado de la población de los "abuelos". En mucho, las remesas de las mujeres son resultado de su participación de la economía del cuidado.

Es importante mencionar cómo el crédito otorgado bajo la influencia de Yunes en forma de microcréditos ha pasado a formar parte de la alternativa para el empoderamiento, principalmente, de las mujeres. Pero quizás más significativo es saber quiénes son las empresas otorgantes de los créditos para los pobres cuyo lenguaje viene a ser parte de la alternativa para empoderar y crear nuevos empresarios. Difícilmente se podría estar de acuerdo cuando se observa cómo las tasas de rentabilidad de estas empresas financieras han hecho a partir del negocio del crédito grandes ganancias. La rentabilidad de estas empresas financieras es mucho mayor a la banca comercial y la banca de desarrollo. El empoderamiento de las mujeres a través del microcrédito ante el desdibujamiento de la banca de desarrollo y de los proyectos estatales es parte del lenguaje para salir de la pobreza. El discurso hegemónico del Banco Mundial y del Banco Interamericano del Desarrollo particularmente en las zonas marginadas de América Latina ha situado al microcrédito como parte de una alternativa para erradicar la pobreza. Discurso que se ha repetido también en zonas marginadas en Asia y África.

Por último, sólo resta mencionar el papel de las migraciones de manera general. El envío de remesas de los trabajadores a sus familias fue cercano a 230 mil millones de dólares en 2010. Si tomamos en cuenta que cada vez son más mujeres las que emigran en busca de mejores oportunidades una parte considerable de estos flujos de capitales forman parte de las familias que se quedan en el país de origen. Son familias, en su mayoría, encabezadas por madres y abuelas que cuidan a los hijos y nietos. Poco porcentaje de las remesas va para proyectos emprendedores ya que en su mayoría son parte del ingreso de consumo del núcleo familiar. 
A continuación presentamos en un esquema la síntesis de la relación entre los cambios macroeconómicos y la microeconomía pasando por las políticas económicas.

Comportamiento en la economía mundial y los mercados internacionales:

De las esferas de la producción, reproducción y financiera

Macroeconomía: crisis económica y financiera

\begin{tabular}{|l|l|lr|lr|}
\hline $\begin{array}{l}\text { Flujos de capital: } \\
\text { grandes ganancias y yuda } \\
\text { contracción de flujos }\end{array}$ & $\begin{array}{l}\text { Ayualial: } \\
\text { volatidad }\end{array}$ & $\begin{array}{l}\text { Remesas: aumento } \\
\text { creciente } \\
\text { disminución }\end{array}$ & $\begin{array}{l}\text { Tipos de cambio: } \\
\text { sobrevaluación y } \\
\text { devaluación }\end{array}$ \\
\hline
\end{tabular}

Comercio, Commodities \& Servicios: exportaciones e importaciones

Mesoeconomía: Programas de Austeridad del FMI y Banco Central

\begin{tabular}{|l|l|l|l|l|l|l|}
\hline D & Gasto & & & & I & \\
I & Público & \multirow{2}{*}{ Créditos } & Exclusión & Capacidad & G & AUMENTO \\
S & Y & DE \\
M & Gasto & & Social & de & R & DESEMPLEO \\
I & Privado & & & consumo & E & Y \\
N & & & & & S & SUBEMPLEO \\
U & & & & & O & \\
C & & & & & & \\
I & & & & & & \\
Ó & & & & & & \\
N & & & & & & \\
\hline
\end{tabular}

\begin{tabular}{|l|l|l|l|l|l|}
\hline $\begin{array}{l}\text { Respuestas de } \\
\text { política }\end{array}$ & $\begin{array}{l}\text { Estímulos } \\
\text { fiscales }\end{array}$ & $\begin{array}{l}\text { Protección } \\
\text { Social }\end{array}$ & Inversiones & $\begin{array}{l}\text { Políticas de } \\
\text { ayuda }\end{array}$ & $\begin{array}{l}\text { Política } \\
\text { laboral }\end{array}$ \\
\hline
\end{tabular}

Microeconomía: Funcionamiento de los Hogares

\begin{tabular}{|l|l|l|l|l|l|}
\hline Microcréditos & $\begin{array}{l}\text { Activos e } \\
\text { Inversiones }\end{array}$ & Consumo & $\begin{array}{l}\text { Capacidad } \\
\text { de trabajo }\end{array}$ & $\begin{array}{l}\text { Economía del } \\
\text { cuidado y la } \\
\text { reproducción }\end{array}$ & $\begin{array}{l}\text { Bienestar } \\
\text { social }\end{array}$ \\
\hline
\end{tabular}

\section{CICLOS ECONÓMICOS Y MERCADO LABORAL FEMENINO EN AMÉRICA LATINA}

El ciclo económico caracterizado por recurrentes crisis económicas y financieras durante los últimos cuarenta años han transformado las pautas de las mujeres como proveedoras de ingresos y como reproductoras de la fuerza de trabajo y del cuidado de la misma. Para Chávez (2010),

“... la globalización y desconcentración de los procesos productivos y de los servicios son elementos importante que han influido en la definición y transformación 
del trabajo femenino. La existencia de abundante mano de obra desempleada y la flexibilidad de las instituciones reguladoras del trabajo en países menos desarrollados, ha proporcionado mano de obra barata a las fábricas del "mercado mundial” de las industrias automotriz, electrónica y de la confección, que si bien crean algunos empleos formales, favorecen de manera directa o indirecta trabajos no formales mediante la subcontratación a pequeñas empresas y microempresas, a trabajadores por cuenta propia, trabajo a domicilio y de producción familiar, que es fundamentalmente femenino (Chávez, 2010:54)”.

En América Latina, las mujeres a principios de los setenta representaban un porcentaje mucho menor de la población económicamente activa en comparación con los hombres. La hipótesis de que las crisis recurrentes en América Latina permitieron una mayor participación de las mujeres en el mercado laboral para proveer ingresos no necesariamente es válida para otras autoras.

“La familia como institución compleja y dinámica enfrenta otra paradoja. Por una parte, es refugio y apoyo frente a condiciones cambiantes que generan inseguridad en el ámbito laboral (desempleo, bajos salarios), de la salud (drogadicción, enfermedad y muerte); de la educación (exclusión) y de la violencia (delictual). Al mismo tiempo, las modificaciones de las familias en el tiempo y el efecto que provocan en ellas tanto las tensiones externas como las relaciones en su propio seno pueden activar fuentes importante de inseguridad interna, como cambios de estado civil (separación, divorcio), migraciones y violencia intrafamiliar. Desde esta perspectiva, la familia es muy vulnerable a las crisis, aunque a la vez constituye la institución más socorrida de protección frente a ellas (Arriagada, 2001: 144)”.

La composición de la familia ha ido paulatinamente cambiando y el número de hogares con jefas de hogar y principales proveedoras del ingreso familiar ha transformado la familia tradicional. Los hijos hoy en día viven con la madre o con el padre o con otros miembros de la familia extensa. Los hogares encabezados por mujeres han tomado fuerza a partir de los setentas. El Estado poco ha avanzado en la formulación del financiamiento en infraestructura para incrementar el apoyo a las mujeres y a la sociedad en seguridad pública, pensiones y educación (Fassler, 2009). Las políticas públicas con perspectiva de género son insuficientes. No obstante, que en los países democráticos las mujeres cumplen visibilidad al representar la otra mitad de las votaciones al menos desde la heterogeneidad que existe en los países de América Latina.

Hay otros procesos que incidieron también en la ampliación del mercado laboral con rostro femenino. Un ejemplo, es el caso de Brasil donde la migración de los hombres a otros lugares se realizó buscando mejores oportunidades de ingresos dejando a las mujeres en sus lugares de origen; ellas iniciaron la emigración para trasladarse con el jefe de familia poco tiempo después y, posteriormente, las mujeres emprendieron el éxodo en busca de mejores oportunidades (Ferreira-Batista y Cacciamali, 2008: 54). En México, “... son cada vez más mujeres las que van en busca de oportunidades de empleo para satisfacer los ingresos de sus familias que 
se quedan en las ciudades y pueblos” (Girón y González Marín, 2009:131). Sin lugar a dudas "la migración ha sido quizás uno de los mayores logros de las políticas de estabilización” y la forma de enfrentar los ciclos económicos por parte de la población no importando la raza, el género y la clase social.

Los procesos sociales y económicos apoyados en las últimas décadas han tejido cambios muy importantes en las condiciones de la mujer y en las oportunidades del empleo. Por ello, se sostiene que no es sólo la incorporación de las mujeres al trabajo lo que ha incidido en la transformación de la familia. A los procesos productivos se tienen que sumar las modificaciones de la composición demográfica; nuevas pautas de consumo y trabajo; acceso masivo pero segmentado de los bienes y servicios sociales (educación, seguridad social y salud); promoción de la libertad social e individual; progreso social para el desarrollo de las potencialidades individuales, vocación democrática; progresiva secularización de la acción colectiva; mayor representación democrática en el gobierno; y, una sociedad multicultural (Arriagada, 2002).

Las políticas de estabilización han impactado a toda la sociedad y cruzan todas las clases sociales. Es importante mencionar que países con crecimiento económico sostenido como son Brasil y Argentina han tenido un mejoramiento de los ingresos de los asalariados. Incluso se reportan menores índices de pobreza durante la última década.

Hay indicadores asimétricos que indican como la situación ha mejorado en los países del Cono Sur y los países que conforman UNASUR contrario a México y Centroamérica. Indudablemente que la cercanía a Estados Unidos y el impacto de la crisis se ha hecho notar con mayor dureza en los países cuya integración económica y comercial es mayor en la región del Tratado de Libre Comercio de América del Norte (TLCAN). Es un hecho que los países cuyo comercio está más relacionado con Europa se verán afectados por los movimientos especulativos que está presentado la Unión Europea.

\section{LAS ESFERAS PÚBLICAS TRASNACIONALES ${ }^{4}$}

Indudablemente, la vida privada es objeto de las políticas públicas no sólo en un país o región sino a nivel mundial. La plataforma de Beijing en 1994 y los Retos

\footnotetext{
4 El concepto de esfera pública trasnacionales no solamente fue desarrollado para entender los flujos de comunicación empíricos sino para contribuir a la teoría de la democracia políticamente normativa. En esta teoría la democracia es concebida como un espacio para la generación de comunicación de opinión pública que otorgue una validez política moral. La fuerza política empodera al ciudadano y lo convierte en una fuerza pública válida que pueda incidir en el Estado. Juntas estas dos ideas de la opinión pública y del empoderamiento ciudadano vis-à-vis el Estado -son esenciales para el concepto de esfera pública en la teoría de la democracia. Sin ellas, el concepto pierde la fuerza crítica y su importancia política (Fraser: 2007).
} 
del Milenio de Naciones Unidas han establecido un carácter específico a los objetivos del desarrollo. En algunos puntos de las metas del milenio la perspectiva de género está presente. Por ello, es muy importante retomar el concepto de esfera pública trasnacional para entender que ante una crisis económica y financiera más profunda que la crisis de 1929 se deben de sustentar políticas públicas nacionales a través de los organismos internacionales para enfrentar el ciclo económico a través del gasto público para erradicar la pobreza, aumentar el gasto social en educación, salud y vivienda creando nuevas oportunidades de trabajo.

La importancia de recoger el concepto de "esfera pública transnacional” de Fraser (2007) radica en la necesidad de una agenda social internacional frente a la determinación de los organismos financieros internacionales, para cambiar una agenda con perspectiva de justicia social internacional.

Es decir, ante las políticas macroeconómicas contraccionistas que se han dirigido a solventar los problemas de la deuda pública y las carteras vencidas de los bancos es muy importante plantear un régimen de conducta social donde la agenda del desarrollo tenga metas bien definidas.

Por ello, es importante tomar los indicadores del Foro Económico Mundial empleados para definir la brecha de género entre los diferentes países. Esto requiere pasar de políticas restrictivas a políticas expansivas. Devolverle al Banco Central su función de empleador de última instancia por arriba de la función de prestamista de última instancia. Es decir, conducir al Banco Central como creador de empleo. Sumar a dicho esfuerzo un gobierno fuerte basado en una política fiscal, monetaria y financiera cuyos objetivos sean un mejor desarrollo de la sociedad. Se tendrá que garantizar la educación y la salud para cerrar la brecha de género a partir de presupuestos públicos con enfoque de género (Villota, Jubeto y Ferrari 2009).

La necesidad de presentar una agenda de desarrollo feminista en el marco de la democracia responde a una nueva realidad económica, política y social. La pregunta es cómo, cuándo y para qué presentarla y dónde manifestarla.

“... ¿cómo mantener la autonomía transgresora de los feminismos en estas nuevas realidades? En estos momentos a diferencia de la década de los ochenta, una autonomía que descanse solo en la defensa del discurso y del espacio propios puede ser muy aislante, como también lo sería una autonomía que no recupere las múltiples interacciones con otros procesos y luchas por la democracia, la ciudadanía desde la sociedad civil, porque lo que se avanza en la defensa propia, sin articulación, no produce transformación de largo aliento” (Vargas, 2008:130).

La articulación entre agenda de desarrollo y alternativas a la crisis con rostro femenino se tienen que cerrar en la articulación de las políticas implementadas desde la macroeconomía pasando por la mesoeconomía hasta llegar a la microeco- 
nomía aterrizada en los hogares. El logro del feminismo lo define Benería en su artículo sobre El Hombre de Davos.

“...el feminismo ha sido muy importante en la lucha por soluciones a nivel descentralizado, local e institucional; ha luchado contra la discriminación y las desigualdades a muchos niveles; ha cambiado procesos institucionales y de toma de decisiones; ha incorporado nuevas agendas en la política de la vida cotidiana; ha influido políticas nacionales; ha tenido impacto en las agendas internacionales; y ha insistido en que el bienestar humano debe ponerse al centro de los debates sobre política económica y social. Ahora también debe ir al encuentro de los desafíos que plantea la globalización” (Benería, 1999).

En este trabajo nosotros agregaríamos que el feminismo debe de enfrentar la crisis económica y la fragilidad financiera con una agenda con perspectiva de género en el ámbito de políticas fiscales, financieras y monetarias. Contrariamente a los programas de estabilización que dañan el rostro femenino del desarrollo.

\section{REFLEXIONES FINALES}

Para concluir este trabajo, es importante retomar la cita de Simone de Beauvoir donde la mujer a través del trabajo se siente “...productiva y activa...tiene noción de trascendencia...y...no necesita un mediador masculino”. Sin embargo, parafraseando las palabras de nuestra autora es importante destacar que no porque la mujer se inserte al mercado laboral y bajo el supuesto de que ha alcanzado a lo largo de un siglo equidad en el trabajo,

“...la maldición que pesa sobre la mujer...consiste en que no se le permite actuar, por lo que se obsesiona en la imposible persecución del ser a través del narcisismo, del amor y la religión”.

El impacto de la crisis en las emociones de los individuos y en su relación con los integrantes de la familia determina variaciones en la conducta del núcleo familiar. Hasta el presente, las emociones y los sentimientos de las familias no han sido estudiadas desde la psicología, economía y sociología desde una visión integral. Quizás uno de los retos desde la academia es buscar los puntos de encuentro entre el mejoramiento de las oportunidades para las mujeres, la satisfacción de sus necesidades, la felicidad como seres humanos y el derecho humano al empleo y la equidad.

Desde una perspectiva de género, el cuestionamiento del modelo de reproducción basado en recursos no renovables que han impacto en dos formas: la primera en el ámbito geopolítico expresado en la conformación de nuevas zonas económicas; segundo, en el cambio climático, ha implicado transformaciones en el proceso de acumulación a lo largo de los últimos setenta años. En este rol del desenvolvimiento del desarrollo capitalista las mujeres han sido envueltas en todos los circuitos del proceso de acumulación capitalista. El feminismo junto con el "neolibera- 
lismo" tiene puntos de acuerdo donde la equidad en el trabajo y la inserción de las mujeres al mercado laboral como símbolo de emancipación jugaron un papel determinante en el empobrecimiento del género femenino.

Hoy, en una situación de constante inestabilidad y fragilidad económica, en el curso de una crisis y una futura estanflación, las mujeres son más dependientes como proveedoras de la vida y del ingreso ante el desdibujamiento de los estados donde el bienestar de la sociedad fue un baluarte alcanzado por la democracia.

\section{BIBLIOGRAFÍA}

ARRIAGADA, I. (2002). "Cambios y Desigualdades en las Familias latinoamericanas”. Revista de la CEPAL, No. 77, agosto, Santiago de Chile, Chile.

http://www.eclac.org/publicaciones/xml/9/19349/lcg2180e Arriagada.pdf

ARRIAGADA, I. (2001). "Familias latinoamericanas. Diagnóstico y políticas públicas en los inicios del nuevo siglo”. División de Desarrollo Social Santiago de Chile, diciembre.

http://www.eclac.org/publicaciones/xml/4/9144/lcl1652-p.pdf

ANTONOPOULOS, R. [2009]. "The Current Economic and Financial Crisis: A Gender Perspective”. Working Paper No. 562, The Levy Economic Institute at Bard College.

BENERÍA, L. (1999). "Mercados Globales, Género y El Hombre de Davos” en CARRASCO, C. (1999). Mujeres y Economía: Nuevas perspectivas para viejos y nuevos problemas. Instituto de la Mujer, Cooperativa El Economista. Icaria editorial, S. A. No. 147. Barcelona, España.

BERGER, S. (2010). "América Latina, la Crisis y el Feminismo. Pensando Junto con Nancy Fraser" en GIRÓN, A. (coordinadora) Crisis económica: una perspectiva feminista desde América Latina. Editorial CLACSO, Instituto de Investigaciones Económicas y Universidad Central de Venezuela.

CARRASCO, C. (1999). Mujeres y Economía: Nuevas perspectivas para viejos y nuevos problemas. Instituto de la Mujer, Cooperativa El Economista. Icaria editorial, S. A. No. 147. Barcelona, España.

CHAVEZ, M. (2010). Trabajo Femenino: las nuevas desigualdades, Instituto de Investigaciones Económicas, Universidad Nacional Autónoma de México.

CROTTY, J. (2000). "Structural Contradictions of the Global Neoliberal Regime", paper presented for "Neoliberalism: Theory and Practice," sponsored by the Union for Radical Political Economics at the Allied Social Science Association meetings in Boston on January 7-9th.

DE BEAUVOIR, S. (1970). "La Mujer Independiente” en El Segundo Sexo: Los hechos y los mitos, Talleres "El Gráfico/Impresores”, Buenos Aires, Argentina.

ELSON, D. [2002]. "International Financial Architecture: A View from the Kitchen", [mimeográfo] para publicación en Política Femina, spring.

http://www.eclac.org/mujer/curso/elson1.pdf 
ELSON, D. (2011). “Gender and the global economic crisis in developing countries: a framework for análisis” en Gender and the Economic Crisis, Practical Action Publishing, Oxfam GB.

FASSLER, C. (2009). Hacia un sistema nacional integrado de cuidados. Ed. Red Género y Familia y Ediciones Trilce, Montevideo.

FERREIRA-BATISTA, N. y CACCIAMALI, M. (2008). "Hiato Salarial entre Homes e Mulheres Migrantes” en A Construção da Gênero e Raça na América Latina Do Século XXI: o Caso do Brasil. São Carlos: Suprema Gráfica e Editora. Brasil.

FINANCIAL TIMES (2011), “Remittances: how funds move around the World”, junio 14, consultado el 8 de agosto. http://www.ft.com/cms/s/0/f422b4a6-95ca-11e08f82-00144feab49a.html\#ixzz1UTuHof5I.

FRASER, N. (2007). “Transnationalizing the Public Sphere, On the Legitimacy and Efficacy of Public Opinion" in a Post-Westphalian World.

http://eipcp.net/transversal/0605/fraser/en

GIRÓN, A. y GONZÁLEZ MARÍN, M. (2009). “Género y Políticas Macroeconómicas: Migración en México” en GIRÓN, A. (coordinadora) Género y Globalización. Colección Grupos de Trabajo. Editorial CLACSO.

GIRÓN, A. (2010). “Los circuitos de la crisis: perspectiva feminista” en Crisis económica: una perspectiva feminista desde América Latina. Editorial CLACSO, Instituto de Investigaciones Económicas y Universidad Central de Venezuela.

http://bibliotecavirtual.clacso.org.ar/ar/libros/grupos/giron.f.pdf

KERR, J. and SWEETMAN, C. (2003). Women Reinventing Globalisation. Oxfam Great Britain.

http://www.scribd.com/doc/52830493/Women-Reinventing-Globalisation

PARDO, M. (2003), "Reseña de programas sociales para la superación de la pobreza en América Latina”. Serie de Estudios estadísticos y prospectivos no. 20. Comisión Económica para AméDivisión de Estadística y proyecciones Económicas. Santiago de Chile, octubre.

http://www.eclac.cl/publicaciones/xml/1/14941/lcl1906e.pdf

PEARSON, R. and SWEETMAN, C. (2011). Gender and the Economic Crisis, Practical Action. Publishing, Oxfam GB.

http://www.oxfam.org.uk/resources/policy/gender/downloads/bk-gender-economic-crisis-100211-en.pdf

POLANYI, K. (20009, La gran transformación. Ediciones Casa Juan Pablo, México, D.F.

ROJAS-SUAREZ, L. (2010). “The International Financial Crisis: Eight Lessons for and from Latin America”. Center for Global Development, Working Paper 2002.

VARGAS, V.y BARRIG, M. (1999). “Una Agenda Feminista: El Rescate de la Utopía” en VARGAS, V. (2008). Feminismo en América Latina: Su aporte a la política ya a la democracia. Colección Transformación Global. Universidad Mayor de San Marcos, Programa Democracia y Transformación Global, Centro de la Mujer Peruana Flora Tristán. Lima, Perú.

VILLOTA, P., JUBETO, Y. y FERRARI I. (2009). Estrategias para la Integración de la Perspectiva de Género en los Presupuestos Públicos. Instituto de la Mujer, Ministerio de la Igualdad. Observatorio 17. Madrid, España.

WORLD ECONOMIC FORUM (2007). The Gender Gap. Switzerland. 
YOUNG, B. (2002). "Financial Crisis in Asia and the Feminization of Human Security" for the panel Constitution and Contradiction in Global Capitalism: Beyond Comparative and International Political Economy, Institute of Political Science, University of Muenster, Germany. New Orleans, March. http://www.ruhr-uni-bochum.de/fiab/pdf/veranstaltungen/young.pdf 